PROCEEDINGS OF THE

AMERICAN MATHEMATICAL SOCIETY

Volume 136, Number 1, January 2008, Pages 257-261

S 0002-9939(07)09060-0

Article electronically published on October 5, 2007

\title{
GLOBAL WELL-POSEDNESS OF DISSIPATIVE QUASI-GEOSTROPHIC EQUATIONS IN CRITICAL SPACES
}

\author{
HANTAEK BAE
}

(Communicated by David S. Tartakoff)

\begin{abstract}
We prove global well-posedness for the dissipative quasi-geostrophic equation with initial data in critical Besov spaces $B_{p, q}^{1+\frac{2}{p}-2 \alpha}, 0<\alpha \leq 1$, provided that the $B_{p, q}^{1+\frac{2}{p}-2 \alpha}$ norm of the initial data is sufficiently small compared with the dissipative coefficient $\kappa$.
\end{abstract}

\section{INTRODUCTION}

We are concerned with the two dimensional dissipative quasi-geostrophic equation

(DQG)

$$
\left\{\begin{array}{l}
\theta_{t}+u \cdot \nabla \theta+\kappa(-\triangle)^{\alpha} \theta=0 \\
u=\left(-R_{2} \theta, R_{1} \theta\right) \\
\theta(x, 0)=\theta_{0}(x)
\end{array}\right.
$$

where the scalar $\theta$ represents the potential temperature, $u$ is the fluid velocity, and $R_{1}, R_{2}$ are the usual Riesz transform. For the physical background of this equation, one may check [1, 3] and references therein for the details. We solve the open problem given by [1]; namely, with $\theta_{0} \in B_{p, q}^{1+\frac{2}{p}-2 \alpha}$, for $1 \leq p, q<\infty$, what is the well-posedness of (DQG)? Two crucial estimates were proved in [3], 4], and we use those estimates to get the following result.

Theorem. There exists a constant $\epsilon_{0}>0$ such that for any $\theta_{0} \in B_{p, q}^{1+\frac{2}{p}-2 \alpha}$ with $\left\|\theta_{0}\right\|_{B_{p, q}^{1+\frac{2}{p}-2 \alpha}}<\epsilon \leq \epsilon_{0}$, (DQG) has a unique global solution $\theta$, which belongs to $C\left([0, \infty) ; B_{p, q}^{1+\frac{2}{p}-2 \alpha}\right)$.

\section{Proof of theorem}

Step 1. A priori estimates. Let $\triangle_{j}$ be the Fourier multiplier given by $\triangle_{j} f=$ $\Phi_{j} * f(j=0, \pm 1, \pm 2, \cdots)$ where $\Phi_{j}(\xi)$ is a smooth function localized around $|\xi|=2^{j}$ satisfying $\sum_{k=-\infty}^{\infty} \Phi_{k}=1$, except for $\xi=0$. Applying the operator $\triangle_{j}$ to the first equation of (DQG), we obtain

$$
\frac{d}{d t} \triangle_{j} \theta+\triangle_{j}(u \cdot \nabla \theta)+\kappa(-\triangle)^{\alpha} \triangle_{j} \theta=0
$$

Received by the editors December 4, 2006.

2000 Mathematics Subject Classification. Primary 35Q40, 75D03. 
Multiplying by $\frac{1}{p} \triangle_{j} \theta \cdot\left|\triangle_{j} \theta\right|^{p-2}$ in the above equation and then integrating with respect to $x$, we have

$$
\begin{gathered}
\frac{d}{d t}\left\|\triangle_{j} \theta\right\|_{L^{p}}^{p}+\kappa \frac{1}{p} \int(-\triangle)^{\alpha} \cdot \triangle_{j} \theta \cdot \triangle_{j} \theta \cdot\left|\triangle_{j} \theta\right|^{p-2} \\
=-\frac{1}{p} \int \triangle_{j}(u \cdot \nabla \theta) \cdot \triangle_{j} \theta \cdot\left|\triangle_{j} \theta\right|^{p-2} .
\end{gathered}
$$

$\mathrm{Wu}[4$ proved the following lower bound estimate:

$$
\begin{gathered}
\int(-\triangle)^{\alpha} \cdot \triangle_{j} \theta \cdot \triangle_{j} \theta \cdot\left|\triangle_{j} \theta\right|^{p-2} \\
\geq C \cdot 2^{2 j \alpha} \cdot\left\|\triangle_{j} \theta\right\|_{L^{p}}^{p} .
\end{gathered}
$$

So we obtain that

$$
\begin{aligned}
& \frac{d}{d t}\left\|\triangle_{j} \theta\right\|_{L^{p}}^{p}+C \cdot \kappa \cdot 2^{2 j \alpha} \cdot\left\|\triangle_{j} \theta\right\|_{L^{p}}^{p} \\
& \quad \leq\left. C \cdot\left|\int \triangle_{j}(u \cdot \nabla \theta) \cdot \triangle_{j} \theta \cdot\right| \triangle_{j} \theta\right|^{p-2} d x \mid .
\end{aligned}
$$

We decompose $(u \cdot \nabla \theta)$ as a paraproduct. (We obtain estimates of this product term. See the appendix.) Then,

$$
\begin{aligned}
& \frac{d}{d t}\left\|\triangle_{j} \theta\right\|_{L^{p}}^{p}+C \cdot \kappa \cdot 2^{2 j \alpha} \cdot\left\|\triangle_{j} \theta\right\|_{L^{p}}^{p} \\
& \quad \leq C \cdot 2^{2 j \alpha} \cdot 2^{-j\left(1+\frac{2}{p}-2 \alpha\right)} \cdot a_{j}\left\|\triangle_{j} \theta\right\|_{L^{p}}^{p-1}\|\theta\|_{B_{p, q}^{1+\frac{2}{p}-2 \alpha}}^{2}
\end{aligned}
$$

Dividing both sides by $\left\|\triangle_{j} \theta\right\|_{L^{p}}^{p-1}$,

$$
\begin{aligned}
& \frac{d}{d t}\left\|\triangle_{j} \theta\right\|_{L^{p}}+C \cdot \kappa \cdot 2^{2 j \alpha} \cdot\left\|\triangle_{j} \theta\right\|_{L^{p}} \\
& \quad \leq C \cdot 2^{2 j \alpha} \cdot 2^{-j\left(1+\frac{2}{p}-2 \alpha\right)} \cdot a_{j}\|\theta\|_{B_{p, q}^{1+\frac{2}{p}-2 \alpha}}^{2} .
\end{aligned}
$$

By solving the above differential equation of time, we get

$$
\begin{aligned}
\left\|\triangle_{j} \theta(t)\right\|_{L^{p}} \leq & e^{-t 2^{2 j \alpha} \kappa} \cdot\left\|\triangle_{j} \theta_{0}\right\|_{L^{p}} \\
& +C \cdot a_{j} \cdot 2^{2 j \alpha} \cdot 2^{-j\left(1+\frac{2}{p}-2 \alpha\right)} \int_{0}^{t} e^{-(t-s) 2^{2 j \alpha} \kappa}\|\theta\|_{B_{p, q}^{1+\frac{2}{p}-2 \alpha}}^{2} .
\end{aligned}
$$

By Young's inequality in time,

$$
\begin{aligned}
& \left\|\triangle_{j} \theta(t)\right\|_{L_{T}^{\infty} L^{p}} \leq\left\|\triangle_{j} \theta_{0}\right\|_{L^{p}} \\
& \quad+\frac{C}{\kappa} \cdot a_{j} \cdot 2^{2 j \alpha} \cdot 2^{-j\left(1+\frac{2}{p}-2 \alpha\right)} \cdot\|\theta\|_{\widetilde{L}_{T}^{\infty} B_{p, q}^{1+\frac{2}{p}-2 \alpha}}^{2} .
\end{aligned}
$$

We note that $\|\theta(t)\|_{L^{p}} \leq\left\|\theta_{0}\right\|_{L^{p}}$ was proved in $[3$. So,

$$
\|\theta\|_{\widetilde{L}_{T}^{\infty} B_{p, q}^{1+\frac{2}{p}-2 \alpha}} \leq\left\|\theta_{0}\right\|_{B_{p, q}^{\frac{2}{p}+1-2 \alpha}}+\frac{C}{\kappa}\|\theta\|_{\widetilde{L}_{T}^{\infty} B_{p, q}^{1+\frac{2}{p}-2 \alpha}}^{2} .
$$


Step 2. Iteration and uniform estimates. Because the bicontinuous constant arising in the above estimate does not depend on time, we will look for a solution $w(x, t)=\theta(x, t)-S_{\alpha}(t) \theta_{0}$, instead of looking for a solution $\theta(x, t)$, where $S_{\alpha}(t) \theta_{0}=$ $e^{-\kappa t(-\triangle)^{\alpha}} \theta_{0} . w(x, t)$ satisfies

$$
\begin{aligned}
& w_{t}+u \cdot \nabla\left(w+S_{\alpha}(t) \theta_{0}\right)+\kappa(-\triangle)^{\alpha} w=0, \\
& u=\left(-R_{2}\left(w+S_{\alpha}(t) \theta_{0}\right), R_{1}\left(w+S_{\alpha}(t) \theta_{0}\right)\right), \\
& w(x, 0)=0 .
\end{aligned}
$$

We define the following sequences:

$$
\begin{aligned}
& w_{t}^{n+1}+u^{n} \cdot \nabla\left(w^{n+1}+S_{\alpha}(t) \theta_{0}\right)+\kappa(-\triangle)^{\alpha} w^{n+1}=0, \\
& u^{n}=\left(-R_{2}\left(w^{n}+S_{\alpha}(t) \theta_{0}\right), R_{1}\left(w^{n}+S_{\alpha}(t) \theta_{0}\right)\right), \\
& w^{n+1}(x, 0)=0 .
\end{aligned}
$$

Similarly to a priori estimates, we have

$$
\begin{aligned}
& \left.\left\|w^{n+1}\right\|\right|_{\widetilde{L}_{T}^{\infty} B_{p, q}^{1+\frac{2}{p}-2 \alpha}} \\
& \quad \leq \frac{C}{\kappa}\left\|w^{n}\right\|_{\widetilde{L}_{T}^{\infty} B_{p, q}^{1+\frac{2}{p}-2 \alpha}} \cdot\left(\left\|w^{n}\right\|_{\widetilde{L}_{T}^{\infty} B_{p, q}^{1+\frac{2}{p}-2 \alpha}}+\left\|S_{\alpha}(t) \theta_{0}\right\|_{\widetilde{L}_{T}^{\infty} B_{p, q}^{1+\frac{2}{p}-2 \alpha}}\right) \\
& \quad \leq \frac{C}{\kappa}\left\|w^{n}\right\|_{\widetilde{L}_{T}^{\infty} B_{p, q}^{1+\frac{2}{p}-2 \alpha}} \cdot\left(\left\|w^{n}\right\|_{\widetilde{L}_{T}^{\infty} B_{p, q}^{1+\frac{2}{p}-2 \alpha}}+\left\|\theta_{0}\right\|_{B_{p, q}^{1+\frac{2}{p}-2 \alpha}}\right) .
\end{aligned}
$$

Let $\epsilon_{0} \leq \frac{\kappa}{4 C}$, and fix $\eta$ such that $\eta<\epsilon_{0}$. If $\left\|\theta_{0}\right\|_{B_{p, q}^{1+\frac{2}{p}-2 \alpha}} \leq \epsilon<\epsilon_{0}$, then $\|\left. w^{n}\right|_{\widetilde{L}_{T}^{\infty} B_{p, q}^{1+\frac{2}{p}-2 \alpha}}$ are uniformly bounded by $\left\|w^{n}\right\|_{\widetilde{L}_{T}^{\infty} B_{p, q}^{1+\frac{2}{p}-2 \alpha}} \leq \eta$.

Step 3. Equations of difference, existence, and uniqueness. Let $\delta w^{n}=$ $w^{n}-w^{n-1}, \delta u^{n}=u^{n}-u^{n-1}$. Then we have the following system of difference equations:

$$
\begin{aligned}
& \delta w_{t}^{n+1}+u^{n} \cdot \nabla \delta w^{n+1}+\kappa(-\triangle)^{\alpha} \delta w^{n+1}+\delta u^{n} \cdot \nabla\left(w^{n}+S_{\alpha}(t) \theta_{0}\right)=0, \\
& u^{n}=\left(-R_{2}\left(w^{n}+S_{\alpha}(t) \theta_{0}\right), R_{1}\left(w^{n}+S_{\alpha}(t) \theta_{0}\right)\right), \delta u^{n}=\left(-R_{2}\left(\delta w^{n}\right), R_{1}\left(\delta w^{n}\right)\right), \\
& \delta w^{n+1}(x, 0)=0 .
\end{aligned}
$$

Then, as before, we get

$$
\begin{aligned}
\left\|\delta w^{n+1}\right\| \|_{\widetilde{L}_{T}^{\infty} B_{p, q}^{1+\frac{2}{p}-2 \alpha}} & \leq \frac{C}{\kappa} \|\left.\delta w^{n}\right|_{\widetilde{L}_{T}^{\infty} B_{p, q}^{1+\frac{2}{p}-2 \alpha}} \cdot\left(\left\|w^{n}\right\|_{\widetilde{L}_{T}^{\infty} B_{p, q}^{1+\frac{2}{p}-2 \alpha}}+\left\|\theta_{0}\right\|_{B_{p, q}^{1+\frac{2}{p}-2 \alpha}}\right) \\
& <\left.\frac{C}{\kappa}\left\|\left.\delta w^{n}\right|_{\widetilde{L}_{T}^{\infty} B_{p, q}^{1+\frac{2}{p}-2 \alpha}} \cdot(\eta+\epsilon)<\frac{1}{2} \cdot\right\| \delta w^{n}\right|_{\widetilde{L}_{T}^{\infty} B_{p, q}^{1+\frac{2}{p}-2 \alpha}} .
\end{aligned}
$$

So, $w^{n}$ converges to $w$ in $L_{T}^{\infty} B_{p, q}^{1+\frac{2}{p}-2 \alpha}$. Furthermore, we can take $\eta$ as small as we want. Hence $w^{n}$ converges to $w$ in $C\left([0, T) ; B_{p, q}^{1+\frac{2}{p}-2 \alpha}\right)$. Uniqueness can be proved similarly. This completes the proof of theorem. 


\section{Appendix}

We decompose $(u \cdot \nabla \theta)$ as a paraproduct:

$$
\begin{aligned}
\triangle_{j}(u \cdot \nabla \theta)= & \sum_{|j-l| \leq N} \triangle_{j}\left(S_{l-1} u \cdot \triangle_{l} \nabla \theta\right)+\sum_{|j-l| \leq N} \triangle_{j}\left(\triangle_{l} u \cdot S_{l-1} \nabla \theta\right) \\
& +\sum_{l \geq j-N} \sum_{|l-m| \leq 1} \triangle_{j}\left(\triangle_{l} u \cdot \triangle_{m} \nabla \theta\right) .
\end{aligned}
$$

So we have three terms to the right-hand side of (1). Motivated by [2], we decompose $I$ defined below as

$$
\begin{aligned}
I= & \left.\sum_{|l-j| \leq N}\left|\int \triangle_{j}\left(S_{l-1} u \cdot \triangle_{l} \nabla \theta\right) \cdot \triangle_{j} \theta \cdot\right| \triangle_{j} \theta\right|^{p-2} d x \mid \\
\leq & \left.\left|\sum_{|l-j| \leq N} \int\left[\triangle_{j}, S_{l-1} u\right] \nabla \triangle_{l} \theta \cdot \triangle_{j} \theta\right| \triangle_{j} \theta\right|^{p-2} \mid \\
& +\left.\left|\sum_{|l-j| \leq N} \int\left(S_{l-1} u-S_{j-1} u\right) \nabla \triangle_{j} \triangle_{l} \theta \cdot \triangle_{j} \theta\right| \triangle_{j} \theta\right|^{p-2} \mid \\
& +\left.\left|\sum_{|l-j| \leq N} \int S_{j-1} u \cdot \nabla \triangle_{j} \triangle_{l} \theta \cdot \triangle_{j} \theta \cdot\right| \triangle_{j} \theta\right|^{p-2} d x \mid \\
= & I_{1}+I_{2}+I_{3} .
\end{aligned}
$$

$I_{3}$ disappears when integrated, by the divergence free condition of $u$. (From now on, we repeatedly use Bernstein's inequalities.) By Hölder inequality,

$$
\begin{aligned}
I_{1} & =\left.\left|\sum_{|l-j| \leq N} \int\left[\triangle_{j}, S_{l-1} u\right] \nabla \triangle_{l} \theta \cdot \triangle_{j} \theta\right| \triangle_{j} \theta\right|^{p-2} \mid \\
& \leq C \cdot\left\|\left[\triangle_{j}, S_{j-1} u\right] \nabla \triangle_{j} \theta\right\|\left\|_{L^{p}} \cdot\right\| \triangle_{j} \theta \|_{L^{p}}^{p-1} \\
& \leq C \cdot 2^{-j}\left\|\nabla S_{j-1} u\right\|_{L^{\infty}}\left\|\nabla \triangle_{j} \theta\right\|_{L^{p}}\left\|\triangle_{j} \theta\right\|_{L^{p}}^{p-1} .
\end{aligned}
$$

But, by the Calderon-Zygmund theorem, we have

$$
\left\|\nabla S_{j-1} u\right\|_{L^{\infty}} \leq C \cdot 2^{2 j \alpha}\|\theta\|_{B_{p, q}^{1+\frac{2}{p}-2 \alpha}} .
$$

Therefore

$$
I_{1} \leq C \cdot 2^{2 j \alpha} \cdot 2^{-j\left(1+\frac{2}{p}-2 \alpha\right)} \cdot a_{j}\left\|\triangle_{j} \theta\right\|_{L^{p}}^{p-1}\|\theta\|_{B_{p, q}^{1+\frac{2}{p}-2 \alpha}}^{2}
$$

where $\left\{a_{j}\right\} \in l^{q}$ such that $\sum_{j \geq-1} a_{j}^{q}=1$. Similarly

$$
\begin{aligned}
I_{2} & \leq C \cdot\left\|\triangle_{j} u\right\|_{L^{\infty}}\left\|\nabla \triangle_{j} \theta\right\|_{L^{p}}\left\|\triangle_{j} \theta\right\|_{L^{p}}^{p-1} \\
& \leq C \cdot 2^{j\left(1+\frac{2}{p}\right)}\left\|\triangle_{j} u\right\|_{L^{p}}\left\|\triangle_{j} \theta\right\|_{L^{p}}^{p} \\
& \leq C \cdot 2^{2 j \alpha} \cdot 2^{-j\left(1+\frac{2}{p}-2 \alpha\right)} \cdot a_{j}\left\|\triangle_{j} \theta\right\|_{L^{p}}^{p-1}\|\theta\|_{B_{p, q}^{1+\frac{2}{p}-2 \alpha}}^{2} .
\end{aligned}
$$


In the same way, we get the estimate for the second term of (1). The third term, denoted by $I I I$, is given by

$$
\begin{aligned}
I I I= & \left.\left|\sum_{l \geq j-N} \sum_{|l-m| \leq 1} \int \triangle_{j}\left(\triangle_{l} u \cdot \triangle_{m} \nabla \theta\right) \triangle_{j} \theta\right| \triangle_{j} \theta\right|^{p-2} \mid \\
\leq & C \cdot \sum_{l \geq j-N}\left\|\triangle_{l} u\right\|_{L^{p}}\left\|\triangle_{l} \theta\right\|_{L^{p}}\left\|\triangle_{j} \theta\right\|_{L^{p}}^{p-2}\left\|\nabla \triangle_{j} \theta\right\|_{L^{\infty}} \\
\leq & C \cdot 2^{j\left(1+\frac{2}{p}\right)} \cdot\left\|\triangle_{j} \theta\right\|_{L^{p}}^{p-1} \sum_{l \geq j-N}\left\|\triangle_{l} \theta\right\|_{L^{p}}^{2} \\
\leq & C \cdot 2^{j\left(1+\frac{2}{p}\right)} \\
& \cdot\left\|\triangle_{j} \theta\right\|_{L^{p}}^{p-1} \sum_{l \geq j-N} 2^{-2 l\left(1+\frac{2+2}{p}-2 \alpha\right)} \\
& \cdot 2^{l\left(1+\frac{2}{p}-2 \alpha\right)}\left\|\triangle_{l} \theta\right\|_{L^{p}} \cdot 2^{l\left(1+\frac{2}{p}-2 \alpha\right)}\left\|\triangle_{l} \theta\right\|_{L^{p}} \\
\leq & C \cdot 2^{2 j \alpha} \cdot 2^{-j\left(1+\frac{2}{p}-2 \alpha\right)} \cdot a_{j}\left\|\triangle_{j} \theta\right\|_{L^{p}}^{p-1}\|\theta\|_{B_{p, q}^{1+\frac{2}{p}-2 \alpha}}^{2} .
\end{aligned}
$$

\section{ACKNOWLEDGMENT}

The author is deeply thankful to Professor Ping Zhang for kind help, suggestions, and encouragement.

\section{REFERENCES}

[1] Chae, D., Lee, J. : Global well-posedness in the super-critical dissipative quasi-geostrophic equations. Comm. Math. Phys. 233(2), 297-311(2003). MR1962043(2004k:76031)

[2] Chemin, J. -Y., Lerner, N. : Flot de champs de vecteurs non lipschitziens et equations de Navier-Stokes. J. Differ. Eq. 122, 314-328(1995). MR1354312 (96h:35153)

[3] Cordoba, A., Cordoba, D. : A maximum principle applied to quasi-geostrophic equations. Comm. Math. Phys. 249(3), 511-528(2004). MR2084005 (2005f:76011)

[4] Wu, J. : Lower bounds for an integral involving fractional Laplacians and the generalized Navier-Stokes equations in Besov Spaces. Comm. Math. Phys. 263, 803-831(2005). MR2211825 (2006k:35225)

Courant Institute of Mathematical Sciences, New York University, 251 Mercer Street, New York, New York, 10012-1185

E-mail address: hantaek@cims.nyu.edu 